\title{
On the Application of Holographic Projection Technology in the Large - scale Sports Performance Arrangement
}

\author{
Yu Na-Hu1a , JinLei $2 b$ * \\ 1. Ji'nan Unviersity, Sports Department Guangzhou 510632 \\ a:Emaill:454922331@qq.com \\ 2.The Student Physical Health Institute of Henan University Kaifeng 475001) \\ bemail:jlei636982@163.com \\ *JinLei is Corresponding Author
}

Key words: the large - scale sports performance,holographic projection technology,sports performance arrangement

\begin{abstract}
This paper analyzes the advantages and trends of holographic projection technology and the characteristics of large sports performance arrangement using the method of literature, aiming at providing new ideas for the performance arrangement,thus facilitating new artistic system of large sports performance in modern times. Results show that the technology of holographic projection gets rid of the tedious steps in the past computer programming,overcomes the design flaws of 3D computer graphic simulation with its unique 360 - degree holographic imaging, and presents a more realistic three - dimensional image whose effect is closer to actual practice. It provides with a better idea of arrangement of large opening and closing ceremonies as well as large sports performances.
\end{abstract}

\section{Concept and status of holographic laser projection}

The holographic laser projection is also named as virtual imaging technology. It applies interference and diffraction principle to record and reproduce the three-dimensional images. Simply speaking, this technology is to record and reproduce the three-dimensional images towards the actual articles. Its development can be divided into two phases. The first phase is the recording of 3D images, which was conquered in 1947. The holographic three-dimensional photograph was invented by Dennis Gabor from Imperial Collage of London University who was then awarded Nobel Prize in Physics in 1971. However, another technical difficulty "reproduction" of the holographic laser projection was solved in 2001. Now, it almost adopts the holographic films with the touching function, which can be interacted with the holographic images. More advanced point is to apply the water mist to carry out the spatial three-dimensional projection. Before 2000, this technology was basically applied in the military and technology, such as the pilots' simulated flight, and spacemen's simulation training. With the constant solving of the technical difficulties, the application of the holographic laser projection has gradually aroused concern in public[1].

At present, the application domain of the holographic laser projection mainly includes the commercial exhibition, special-effect film making, large opening ceremony and closing ceremony, large-scale art party, large-scale travel party, concert, award ceremony, commercial performance, T-stage fashion show, etc. That the holographic laser projection actually entered into the large-scale party started after the opening and closing ceremonies of Beijing Olympic Games in 2008. It has initiated such technology in the stage. Compared with the foreign countries, it is mainly applied in the concert, large-scale parties and large-scale closing ceremonies. The application of the holographic laser projection is the presentation of the stage effect. With the constant development of the science, the integration of the art and technology has started to become diversified. At the 
same time of making the life better, the technology also brings about more advanced artistic enjoyment. Therefore, the large-scale sports events and performance have started to attract others' attention. Their urgent expectation towards the unknown results of the competitive sports will bring great motivation to the launching of the large-scale competitive events and sports performance. For the director, the good art presentation depends on the arrangement. Good arrangement brings great art charisma and picture motivation sense. Such technology can bring sudden visual impact and spiritual shock, fantastic three-dimensional sense with real and virtual feature, and interesting multi-angle appreciation experience to the audiences. From the research on the technology, it is basically applied in the stage art and choreography but not applied in the dance teaching. Its advantage will bring artistic re-conception to the directors. At the same time of improving the stage effect, it can be applied in the creation of the actors on the stage based on the convenience and three-dimensional effect of the holographic laser project. No matter for the action rehearsal or the arrangement change, there will be new reform[2].

\section{Characteristics and methods of the large-scale sports performance arrangement}

Characteristics of the large-scale sports performance arrangement

Generally, some small-scale sports performance and physical dance mainly take the following aspects into consideration during the artistic creation: the theme of the artistic theme, whether the dance movement is suitable for the theme, whether the arrangement change can be presented, whether the actors perform properly, whether the lighting effect of the stage can apply colors to the atmosphere, etc. however, aiming at the large-scale sports performance, the director should pay attention to the presentation effect of the performance on the stage, the influence of the stage size in the arrangement, whether the distance between the stage and audience has limitation on the performance elements and forms, whether the technical means show clear effect on the background of the art theme, whether the program connecting time and method are reasonable, whether the clothes style and color of the performers can be presented, etc. Therefore, the main characteristics of the large-scale sports performance arrangement are as follows:

(1) Consider the distance and angle between the stage and audience, and the stage background;

(2) Consider the stage size, distance between the stage and audience, and performance arrangement change and form;

(3) Consider the presentation of the actors' clothes and color on the whole arrangement effect;

(4) Apply the scientific and technological means to set off by contrast, and control the smoothness of the program connecting.

Method of large-scale sports performance arrangement

On the stage design, the traditional sports performance arrangement basically adopts the background curtain and the real tools. With regard to the arrangement of the performance content, firstly, proceed with the teaching of the single movement, and then whole lineup design, music selection and editing, and finally whole rehearsal and modification improvement. The traditional arrangement means takes much labor and shows complex working steps. The current large-scale sports performance arrangement method is as follows:

(1) Adopt LED and computer to design the stage background and environment;

(2) The directors should provide constant training and improvement on the performance lineup and dance movement according to the computer and actual training means.

(3) Adopt the following way to do the musical composition: compose the complete movement and then compose the music; select and edit music and then compose the music; select the music, arrange the movement, and then modify the music according to the problems in the arrangement, and make constant improvement. All arrangement methods above start from the front view of the stage. It does not take the visual effect of the large-scale sports performance stage from different angles. The stage background only shows single graphic design or graphic $3 \mathrm{D}$ effect. It shows high requirement on the actors. They should seek for good stage position to get suitable situation for the background. The holographic laser projection takes the real 3D projection as the background and tool, which not provides more actual images for the stage design, but also enables the actors to be 
clear about the stage position and the movement arrangement. All artistic effects are presented through the actors' performance. The technical effect only plays a foiling role. According to the application of the current holographic laser projection technology, the virtual imaging technique has already been improved. The imaging can be the person and thing, such as several virtual images of Jay Chou in his "The Era" Concert, the perfect combination of Beyonce and 3D holographic imaging in the Music Billboard award party in America, virtual singers of Japanese "Hatsune Miku", the holographic laser imaging of India Pavilion in Shanghai World Expo, etc. The imaging can be zoomed in or zoomed out. Besides, the imaging can also been interacted. The simulation training of American military was applied in mid 1920s at the earliest. It was mainly applied in the air force flight training. Therefore, it is feasible to apply the holographic laser projection technology into the stage lineup and actors' movement arrangement.

\section{Advantages of applying the holographic laser projection in the large-scale sports performance arrangement}

Advantage of applying the holographic laser projection in the stage background design

The traditional stage design is to consider the actor's performance and apply what stage background to set off the performance effect according to the artistic theme. Most stage background design is carried out through the computer and LED display screen. For the audiences in the front-side stage, the stage effect is so brilliant. However, for the audiences in the corner, the stage effect cannot be displayed. The holographic laser projection is divided into $180^{\circ}$ holographic projection, $360^{\circ}$ holographic projection and spectra vision. $180^{\circ}$ holographic projection is applicable to the display of single face. $360^{\circ}$ holographic projection is applicable to the opening and closing ceremonies of the large-scale sports meetings. Besides, it is feasible to see 3D images from four sides, which is artistic enjoyment for the audiences from each side near the large-scale stage. Based on $360^{\circ}$ holographic projection, the director can project the actors to the stage, and select the best stage artistic effect that is closest to the actual performance through constantly changeable stage background. The application of the holographic laser projection in the stage design not only can generate three-dimensional spatial imagination, but also can generate interaction between the imagination and actors, and then complete the performance and arouse shocking performance effect. Take a fantastic theatre named Animation Master Norman as an example, the stage art and the filmstrip present extraordinary blending in the space, and show the latest result of the global multi-media art to the audiences. [3]

Advantages of holographic laser projection in the large-scale sports dance arrangement

The large-scale sports dance this paper studies is mainly aimed at the sport performance on Olympic Games, Asian Games, National Athletic Meet, and other large-scale competition. Generally, the directors firstly arrange the individual movement when designing the dance movement, and then teach movement through one to many. With regard to the lineup arrangement, most directors adopt the drawing. The lineup effect mostly lies in the directors' ability of spatial thinking. Stimulate the proposal and screening of the arrangement through the computer. Finally, apply it to the actual drilling of the actors. According to the actors' movement and lineup, carry out constant modification and improvement in the actual drilling. Such arrangement method is relatively complex. It is necessary to think about the drawing, and then do drilling through the computer. After drilling, constantly do modification in. The holographic laser projection can reduce the directors' workload. They can compose the movement by themselves firstly, select proper costume, carry out the holographic recording for the dance movement, project own images to the stage according to the number of people in the large-scale dance as well as the lineup requirements, and then modify the dance movement to make it connect with the lineup change through the visual analysis. Besides, it is also feasible to identify the disadvantages of the lineup arrangement from multiple angles. If it is only the lineup arrangement, it is feasible to zoom in the recorded holographic images and project it to the simulated small stage for visual analysis, which brings good news to the large-scale sports performance arrangement[4]. 
The holographic laser projection is favorable for the actors and dancers to well interpret the artistic theme and effect.

During the rehearsal, as for the traditional large-scale sports performance, it is difficult for the actors to directly imagine the directors' thought and pictures. So, the time, labor and material consumed during the training are really large. Generally, for a large-scale sports performance, especially like the opening and closing ceremonies in Beijing Olympic Games in 2008, each program should go through long-term training and repeated rehearsal, so that it can reach the artistic effect. As for the opening and closing ceremonies of the large-scale sports events, due to the heavy workload, it is necessary to consider the actual effect of the actual stage performance during the design, and connect the directors and actors through the interphone. The workload is so heavy and the actors are not able to directly imagine the directors' design effect. To apply the holographic laser projection to do the rehearsal can project the images and stage background designed by the directors to the large-scale stage for virtual presentation, which is favorable for the actors and dancers to do the drilling after better understanding the directors' intention, and also interpret the theme and effect of the stage art.

\section{Conclusion}

The holographic laser projection technology gets rid of the complex steps of the previous computer programming simulation design, and overcomes the defects of the computer graphic 3D simulation design with the unique $360^{\circ}$ holographic imagining, which forms more real three-dimensional images, and get stage effect closer to the actual rehearsal, exceeds the previous rigid arrangement method. It provides better arrangement technology and innovation for the sports performance of the large-scale competition events, and large-scale closing and opening ceremonies. Its technology avoids the directors' complex arrangement on the computer. The directors can present the arranged holographic images to the actors that participate in the training, and avoid the disadvantages during the actors' training, such as low efficiency and incapability of following the directors' design.

Although the application of the holographic laser projection technology has great sense of image and visual impact, the equipment for this technology is relatively expensive[5]. For the small stage and the sports performance that few people attend, to apply the holographic laser projection technology is not affordable for some ordinary units and performance companies. That is the reason why this paper only studies the large-scale sports performance arrangement. However, with the rapid development of the science, the large-scale sports performance has constantly present diversified, opening and innovative tendency in the inheritance and innovation, which makes the application of the holographic laser projection technology become necessary in the large-scale sports performance arrangement. With the constant appearance of the new technology and new material, the equipment will appear in many domains, such as military, education, entertainment, scientific research, medicine, aviation, communication, academic, etc. What cannot be ignored is that the application of such technology in the large-scale sports performance is not the simple combination of "technology" and "art", instead, it is the effect better than "technology" and "art". Therefore, to apply such technology into the large-scale sports performance arrangement requires our deep understanding and research, and then accelerates the propagation of the Olympic spirit, and forms modern large-scale sports performance art system. For the scientists, the hologram is the reflection of the interaction between the laser beam on the photosensitizer plated with the mercurating and silver compound. For the artists, the hologram has great sense of beauty. It exceeds simple visual depth imagination, and provides the media of recording the three-dimensional information on the two-dimensional plane. However, although people have different viewpoints, there is still some common that the creator of the hologram and the observer are quite exciting. 


\section{References}

[1] Chen Min, Xu Xiaoqin. Comparative Research on Competitive, Body-building and Recreational Sports Show [J]. Notification for Sports Scientific Literature, 2013 (1): 10, 19.

[2] Ma Hongtao, Li Min. Discussion on Sports Performance Art Concept and Classification [j]. Journal of Beijing Sports University, 2006 (9): 1265 - 1267.

[3] Xu Xiuwen, Bo Jianye, Yang Min, Hao Yong. Discussion on 3D, Holographic and Virtual Reality Technology [J]. China's Educational Information. 2011 (13): 85-86.

[4] Chen Jian, Yao Songping. Application of Virtual Reality Technology in Sports Technology Simulation [J]. Sports Science: 2006 (9): 34 - 39.

[5] Chen Shude. Research on Sports Dancing Team Arrangement [J]. Sports \& Science. 2000 (5): 47-49. 